\title{
EFICIÊNCIA DO MODELO CERES-MAIZE NA PREDIÇÃO DA PRODUTIVIDADE DE MILHO EM ÁREAS COM PRESÉNÇA DE VARIABILIDADE ESPACIAL
}

\author{
Ceres-Maize model efficiency in corn yield prediction within spatial variable areas
}

\author{
Sandro Manuel Carmelino Hurtado¹, Carlos Maurício Paglis², Renzo Garcia Von Pinho ${ }^{3}$
}

\begin{abstract}
RESUMO
Modelos são utilizados para a simulação do crescimento de plantas como ferramentas na otimização das práticas de manejo, bem como para estimar produtividades. Com este estudo objetivou-se testar a eficiência do modelo CERES-maize na estimação da produtividade de milho, em função da variabilidade espacial presente na área. Foram instaladas no Campus da UFLA parcelas experimentais em áreas variáveis segundo o teor de saturação por bases (V\%), ao ter-se mostrado decisivo nas observações realizadas. Também foram obtidos dados de temperaturas máxima e mínima, precipitação e radiação solar; dados de solo nas camadas de 0-27, 2745, 45-68 68-80 e 80-100 cm; dados de manejo da cultura do milho, e estimados os parâmetros genéticos do híbrido utilizado. Os resultados da simulação mostraram que as produtividades foram maiores em áreas com elevada saturação por bases, coincidindo com as produtividades observadas. Melhores aproximações nas respostas estimadas ficaram evidentes quando foram utilizados os valores dos parâmetros genéticos próprios a cada parcela. Concluiu-se que o modelo CERES-maize foi capaz de estimar a tendência das diferentes produtividades observadas, em função da variabilidade espacial da saturação por bases (V\%), obtendo-se simulações mais precisas ao utilizar os valores dos parâmetros genéticos próprios a cada parcela.
\end{abstract}

Termos para indexação: Modelagem, DSSAT, Zea mays L.

\begin{abstract}
Simulation models are tools utilized for optimization of managements practices as well as to estimate crop yield. The present study aimed to test the efficiency of the CERES-maize model to simulate corn yield related to the field spatial variability. The experimental area was located at Federal University of Lavras, where experimental plots were established according to the variable areas of base saturation (V\%), a parameter regarded as decisive in the observed yields. Data of maximum and minimum temperatures, rainfall and solar radiation; soil data in the $0-27,27-45,45-68,68-80$ and 80-100 cm layers to each experimental plot, management information of corn crop and genetic parameters of the corn hybrid, were collected. The simulation presented better results when the genetic parameters, particular to each plot, were utilized. Observed yield simulations were higher in areas of elevated base saturation. Due to that, it follows that the simulation was capable to estimate a trend of the distinguished yields as related to the spatial variables of the soil attribute (V\%), obtaining more precise simulations with the use of the values of the genetic parameters estimated in each plot.
\end{abstract}

Index terms: Models, DSSAT, Zea mays L.

(Recebido para publicação em 7 de junho de 2004 e aprovado em 6 de junho de 2005)

\section{INTRODUÇÃO}

O milho pode desenvolver-se sob amplas condições de solo e clima. Em função disso, os produtores tomam decisões em alguns aspectos, tais como, a data de semeadura e taxas de adubações, com a finalidade de obter os máximos lucros e reduzir os riscos associados às imprecisões climáticas (KINIRY et al., 1997).

Por sua vez, a modelagem de culturas, trata de compreender as interações dos componentes do sistema sob estudo, o que, do ponto de vista agronômico, refere-se ao sistema solo-plantaatmosfera. Nesse sentido, a simulação por meio de modelos de culturas é útil na otimização das práticas de manejo, ao prover formas quantitativas de estimar a produtividade das culturas sob diferentes ambientes e climas (XIE et al., 2001).

Entre as vantagens de uso dos modelos de simulação, encontra-se a possibilidade de economizar tempo, esforço e recursos necessários para a tomada de decisões relacionadas ao manejo das culturas. De maneira similar, experimentos de campo detalhados e de longa duração podem ser melhor definidos, quando os modelos proporcionam alcances iniciais nas respostas esperadas (JAME \& CURTFOURTH, 1996). Já, uma das suas principais desvantagens é a grande quantidade de dados necessária para a sua execução, dado o grau de detalhe que apresentam.

\footnotetext{
${ }^{1}$ Engenheiro Agrônomo MSc. em Fitotecnia - Departamento de Agricultura da Universidade Federal de Lavras/UFLA - Cx. P. 3037 - 37.200-000 Lavras, MG - sandrobat@hotmail.com

${ }^{2}$ Ph.D. Professor Adjunto do Departamento de Agricultura/UFLA- Cx. P. 3037 - 37.200-000 - Lavras, MG.

${ }^{3}$ Dr. Professor Adjunto do Departamento de Agricultura/ UFLA - Cx. P. 3037 - 37.200-000 - Lavras, MG.
} 
Um dos programas computacionais que envolvem modelos de culturas é o Decision Support System for Agrotechnology Transfer (DSSAT), considerado importante nas predições do desenvolvimento da cultura sob condições de água e solo variáveis (NIELSEN, 2002).

Dentro do DSSAT, o modelo Crop Environment Resource Synthesis para a cultura de milho (CERES-maize) (JONES \& KINIRY, 1986), simula o crescimento e desenvolvimento do milho, balanço hídrico e níveis de nitrogênio, baseado em quatro variáveis de entrada: solo, clima, manejo do cultivo e genótipos (BOULLON et al., 1996, citados por SOLER, 2000).

De forma geral, os modelos de simulação de crescimento e desenvolvimento das culturas foram projetados para estimar valores em áreas homogêneas. Entretanto, existe variabilidade nas produtividades, a mesma que se explica por fatores como: a densidade da população, estresses hídricos, fatores nutricionais e físicos do solo ou até induzidos pelo próprio manejo, mudando espacialmente dentro da área (BATCHELOR et al., 2002).

A respeito disso, a aplicação de modelos de simulação na predição de produtividades em áreas espacialmente variáveis converte-se em um novo desafio para a pesquisa agrícola. Trabalhos desenvolvidos por Fraisse et al. (2001) e Irmak et al. (2001), tem mostrado que modelos do DSSAT podem estimar de forma adequada as produtividades, quando amostragens adequadas são realizadas em áreas com presença de variabilidade espacial do solo.

Por sua vez, uma forma de superar as limitações espaciais dos modelos de simulação, de acordo com Batchelor et al. (2002), é por meio de simulações em áreas pequenas e homogêneas ao interior das áreas experimentais. Em razão disso, com o presente estudo, objetivou-se testar a eficiência do CERES-maize na simulação da produtividade de milho em áreas espacialmente variáveis, segundo o nível de saturação por bases $(\mathrm{V} \%)$, por ter sido um atributo determinante na produtividade, assim como, testar a interferência da utilização dos valores médios dos parâmetros genéticos nas estimativas realizadas.

\section{MATERIAL E MÉTODOS}

A área de estudo localiza-se no Campus da Universidade Federal de Lavras, Lavras, MG. Apresenta uma área de 0,72 ha, em Latossolo Vermelho, tendo sido cultivada há mais de dez anos com a cultura de milho em sistema convencional. A mesma vem sendo tratada de forma homogênea, embora apresente produções heterogêneas em diferentes zonas do talhão.

A área experimental foi dividida numa malha regular com espaçamentos de $30 \mathrm{~m}$ entre pontos ao longo de três transetos paralelos de $360 \mathrm{~m}$ de comprimento, distanciados $10 \mathrm{~m}$ entre si. Em cada ponto da malha foram retiradas amostras de solo na camada de $0-0,2 \mathrm{~m}$. Após a análise de fertilidade, foram determinados os semivariogramas e gerados os mapas para os diferentes parâmetros do solo, por meio da interpolação por krigagem. Foram determinadas duas áreas de manejo (A e B), diferenciadas pelo nível de saturação por bases (V\%), por ter sido o parâmetro mais representativo para fins da estimativa da produtividade. Instalaram-se, assim, quatro parcelas experimentais, sendo duas em cada área diferenciada, com dimensões de $24 \mathrm{~m} \mathrm{x}$ 4,8 m (Figura 1). Dados de altura de plantas foram determinadas nos mesmos pontos amostrais, gerando posteriormente o mapa de distribuição espacial.

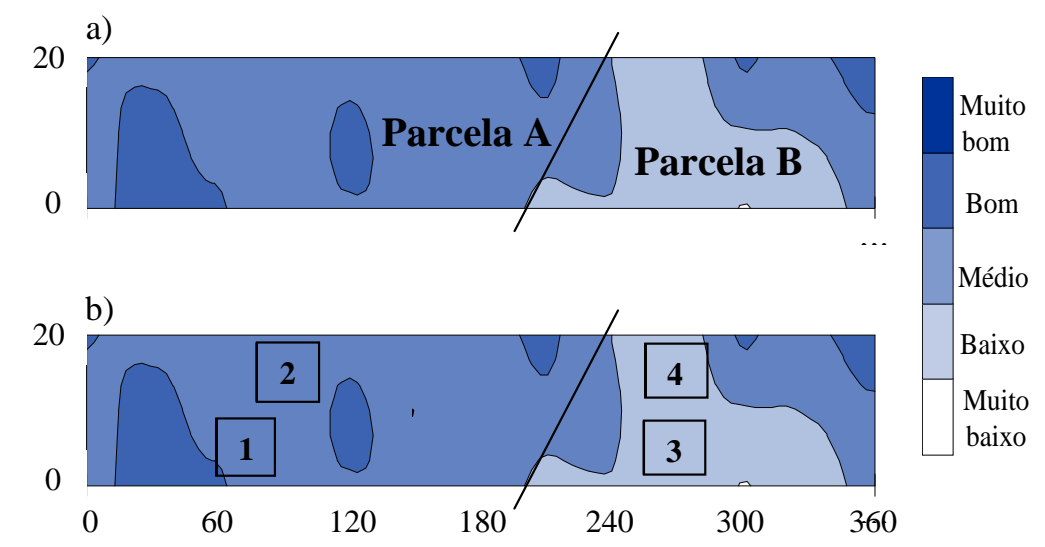

FIGURA 1 - Localização das parcelas experimentais: a) considerando as duas áreas diferenciadas segundo V\%, e b) considerando duas parcelas por cada subárea diferenciada de V\%.

Ciênc. agrotec., Lavras, v. 29, n. 6, p. 1153-1160, nov./dez., 2005 
Sob sistema de plantio convencional, foi semeado o milho híbrido simples A2345 com espaçamento de $0,8 \mathrm{~m}$ entre linhas, objetivando uma população final de 55.000 plantas por hectare. De acordo com as estimativas para o Estado de Minas Gerais (CFSEMG, 1999), não foi necessária a aplicação de calcário. A fertilização contemplou a aplicação de $400 \mathrm{~kg}$ da fórmula 8-28-16 + 0,5\% Zn na adubação de plantio e 300 $\mathrm{kg}$ da fórmula 30-0-20, em cobertura única, no período de emergência da $4^{\mathrm{a}}$ a $6^{\mathrm{a}}$ folha. A colheita foi realizada após a maturidade fisiológica dos grãos e o peso total de grãos, estimado em $\mathrm{kg} \mathrm{ha}^{-1}$, corrigido para $15,5 \%$ de umidade.

Dentre os parâmetros de entrada do modelo de simulação, os climáticos foram determinados com dados diários referentes às temperaturas máxima e mínima $\left({ }^{\circ} \mathrm{C}\right)$, precipitação $\left(\mathrm{mm} \mathrm{dia}^{-1}\right)$ e radiação solar $\left(\mathrm{Mj} \mathrm{m}^{-2} \operatorname{dia}^{-1}\right)$, obtidos da estação meteorológica do Campus, localizada a $100 \mathrm{~m}$ do local experimental, nas épocas correspondentes ao período experimental.

Os dados referentes ao arquivo solo foram obtidos mediante coleta de amostras nas profundidades de $0-0,27$; $0,27-0,45 ; 0,45-0,68 ; 0,68-0,80$ e $0,80-1,00 \mathrm{~m}$, para cada uma das parcelas experimentais. Entre esses dados, o valor de albedo, igual a 0,14 , foi calculado segundo o teor de matéria orgânica do solo, sendo representativo da área estudada. O coeficiente de evaporação na camada superficial (U), estimado segundo a textura da camada do topo do solo, foi de $9 \mathrm{~mm}$, em função de sua textura argilosa (LIMA, 1995). Já na estimativa do parâmetro escoamento superficial, o modelo utilizou o método do número de curva (CN2), sendo este de 89 ao apresentar a área, fileiras simples e boas condições hidrológicas (JONES \& KINIRY, 1986).

$\mathrm{O}$ coeficiente de drenagem (B), determinado em função da porosidade (Po) e umidade na capacidade de campo $(\theta \mathrm{cc})$, foi obtido pelo menor valor para todas as camadas. Uma outra variável considerada foi o fator de peso para a distribuição das raízes (WR), usado para estimar o crescimento relativo de raízes nas camadas do solo onde realmente ocorrem.

Os parâmetros genéticos referentes ao comportamento do híbrido utilizado foram: P1: graus-dia entre a emergência das plantas e o final do estádio juvenil; P2: coeficiente de sensitividade ao fotoperíodo; P5: grausdia entre o florescimento e o ponto de maturidade fisiológica (PMF); G2: número potencial de grãos por planta; e G3: taxa potencial de crescimento de grãos. Para seu cálculo foram realizadas medições do número de grãos por $\mathrm{m}^{2}$, número de grãos por espiga e curva de enchimento de grãos, e determinada a data de florescimento, com o objetivo de comparar esses dados com as estimativas realizadas pela simulação.

Cabe ressaltar que o coeficiente de sensitividade ao fotoperíodo foi assumido como sendo igual a 0,5 , por ser um valor típico para híbridos tropicais (JONES \& KINIRY, 1986). Por sua vez, o parâmetro G3, foi obtido por meio da regressão linear entre o peso da matéria seca dos grãos e os dias após a emergência dos mesmos (mg grãos ${ }^{-1}$ dia $^{-1}$ ) (LIMA, 1995).

Na obtenção dos parâmetros de manejo, foram determinados: a população de plantas (plantas $\mathrm{m}^{-2}$ ), data e profundidade de semeadura $(\mathrm{m})$, índice de área foliar máximo, peso seco no PMF (kg), fitomassa máxima acima do solo no PMF $(\mathrm{kg})$, rendimento de grãos a $15,5 \%$ de umidade $\left(\mathrm{kg} \mathrm{ha}^{-1}\right)$ e latitude do local. É importante destacar que a população de plantas $\mathrm{m}^{-2}$ variou de 5,2 a 5,7 plantas $\mathrm{m}^{-2}$.

No presente experimento, foi realizada a calibração dos parâmetros genéticos, com a finalidade de ajustá-los às condições experimentais, considerando os valores médios dos parâmetros genéticos das quatro parcelas experimentais (Figura 1b), como representativos da área experimental.

Na calibração, o parâmetro P2 foi mantido igual a 0,5. O valor do P1 foi ajustado até as datas estimadas de florescimento ficarem próximas das observadas. Com o novo valor de P1 constante, ajustou-se P5 até as datas estimadas e observadas do PMF, ficarem próximas. No ajuste de G2 foram acrescentados 10 grãos por vez. Finalmente, foi alterado o valor de $\mathrm{G} 3$ a cada $0,01 \mathrm{mg}$ grãos $^{-1} \mathrm{dia}^{-1}$, até o rendimento simulado ficar o mais próximo do observado. Para G2 e G3 considerou-se como erro máximo os valores de $2 \%$ e $1 \%$, respectivamente (LIMA, 1995).

Assim, foram realizadas um total de quatro simulações, duas prévias e duas posteriores à citada calibração. Tanto nas simulações prévias, quanto nas posteriores, considerou-se uma simulação utilizando os valores médios dos parâmetros genéticos das quatro parcelas experimentais, e uma outra, utilizando os valores dos mencionados parâmetros próprios a cada parcela experimental.

Efetuaram-se simulações de produtividade de milho nas áreas diferenciadas segundo o teor de saturação por bases (Figura 1a), assim como, nas áreas correspondentes a cada uma das quatro parcelas experimentais (Figura 1b). Em cada caso, foram utilizados os dados referentes aos arquivos solo e manejo correspondente a cada área. 


\section{RESULTADOS E DISCUSSÃO}

As temperaturas máxima e mínima do ar encontraram-se dentro do padrão normal para as condições de Lavras, não superando as temperaturas médias os $26^{\circ} \mathrm{C}$, o que é desejável às fases de florescimento e maturação (FANCELLI, 2003).

$\mathrm{Na}$ distribuição das precipitações notou-se irregularidades durante o florescimento, o que influenciou a produção final, pela ausência de chuva por um período maior que 14 dias logo após a floração. O período crítico para a cultura de milho em relação ao déficit hídrico situase entre os 15 dias anteriores e posteriores ao florescimento, podendo as quedas na produção, atingir valores de até 4050\% (FANCELLI, 2003). Ribeiro (1998) por sua vez, destaca as quedas na região de Minas Gerais como devidas à expressiva variação da precipitação nos meses de dezembro e janeiro.

A radiação solar apresentou também quedas nas datas próximas ao período de floração (60 d.a.s.), podendo ter influenciado na produção final de grãos por apresentar, esta cultura, uma maior sensibilidade à nebulosidade entre os 10 a 15 dias após o florescimento. Andrade et al. (2000) mencionam a interceptação da radiação fotossinteticamente ativa, nos 15 dias prévios e posteriores a data de floração, como determinante no número de grãos e grãos apicais da espiga.

\section{Estimativa da produtividade prévia à calibração}

Considerando os valores médios dos parâmetros genéticos, simularam-se as produtividades nas áreas diferenciadas segundo níveis de V\% (Figura 1a), assim como, em cada uma das parcelas experimentais (Figura 1b), mantendo os parâmetros de solo e de manejo próprios a cada área (Tabela 1).

As simulações realizadas nas parcelas A e B, diferenciadas segundo o nível de V\% (Figura 1a), evidenciaram a tendência presente nos dados observados, porém, com valores muito próximos entre as parcelas. Este fato pode ser explicado ao ter-se considerado o valor médio dos parâmetros genéticos nas estimativas. Embora seja pequena a diferença entre os dados estimados, a simulação mostra-se efetiva na predição de produtividades em áreas espacialmente variáveis.

De forma análoga, a simulação detectou diferenças entre as produtividades das quatro parcelas experimentais, embora exista uma proximidade nas respostas estimadas. As menores produtividades ocorreram nas parcelas 1 e 3, o que pode ser explicado, por algum fator influenciando a variação de alturas de plantas (Figura 2).

TABELA 1 - Produtividades estimadas e observadas nas diferentes áreas experimentais, considerando os valores médios dos parâmetros genéticos do híbrido A2345.

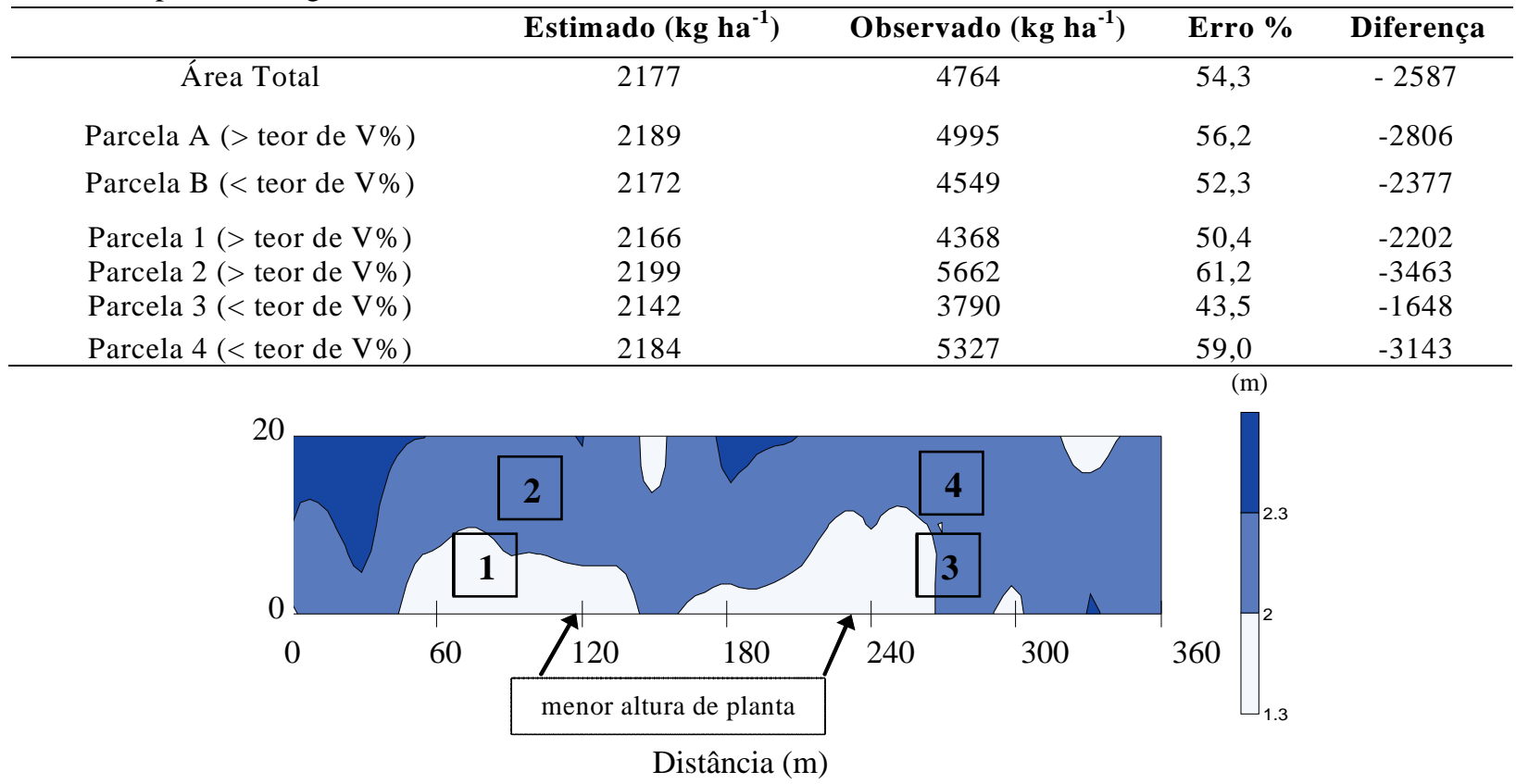

FIGURA 2 - Variabilidade espacial da altura de plantas influenciando a localização das parcelas experimentais. 
Considerando os valores dos parâmetros genéticos próprios a cada parcela (Tabela 2), observa-se nas simulações, uma maior diferença nos valores de produtividade para as parcelas diferenciadas segundo o teor de V\% (Tabela 3). Tal diferença é explicada pela eliminação da influência negativa ao se utilizar os valores médios dos referidos parâmetros genéticos.

Observa-se também que, os erros existentes entre os valores estimados e observados nas parcelas A e B são muito próximos, evidenciando o melhor ajuste do modelo na simulação das produtividades para ambas as parcelas.

Já, nas simulações para cada uma das quatro parcelas experimentais, verificou-se também o maior afastamento entre os valores estimados, em relação aos valores anteriormente simulados,com erros entre valores estimados e observados muito próximos. Corrobora-se, assim, a boa predição das produtividades em função das variações espaciais dos atributos do solo.

Para efeitos de validação do modelo, consistente na comparação das respostas estimadas com as observadas no campo, utilizaram-se os parâmetros genéticos do híbrido A2345 calibrados para as condições experimentais (Tabela 4).

Estimativa da produtividade após à calibração

Calibrado o modelo, realizaram-se simulações considerando a média dos parâmetros genéticos. Esta constatou a permanência da tendência nas estimativas das parcelas A e B (Tabela 5), tal como evidenciado anteriormente (Tabela 1), mostrando valores estimados próximos entre as parcelas. Isto pode ser explicado ao considerar diferenças localizadas só para os dados do arquivo solo e manejo.

Os parâmetros genéticos podem ser influenciados de maneira localizada em aspectos como: a população de plantas, afetando o parâmetro G2, ou a velocidade do acúmulo de matéria seca, afetando o parâmetro G3. Isto se reflete também nas diferentes áreas segundo o teor de saturação por bases, com valores de G3 maiores na parcela A (5,23 $\left.\mathrm{mg}_{\text {grãos }}{ }^{-1} \mathrm{dia}^{-1}\right)$ (Tabela 2).

Simulações realizadas nas quatro parcelas experimentais detectam, de maneira análoga às simulações prévias à calibração, maiores produtividades nas parcelas 2 e 4 . Evidencia-se também um menor erro entre os valores estimados e observados.

Nas simulações considerando os valores dos parâmetros genéticos próprios a cada parcela, observa-se o mesmo valor para os parâmetros P1, P2 e P5 (Tabela 6). Isto em função das datas estimadas do P1 e P5 serem calculadas considerando os graus dia.

TABELA2 - Valores dos parâmetros genéticos correspondentes às diferentes parcelas experimentais, obtidos antes da calibração.

\begin{tabular}{ccccccc}
\hline Parâmetros & Parcela 1 & Parcela 2 & Parcela 3 & Parcela 4 & Parcela A & Parcela B \\
\hline P1 & 300 & 300 & 300 & 300 & 300 & 300 \\
P2 & 0,5 & 0,5 & 0,5 & 0,5 & 0,5 & 0,5 \\
P5 & 720,8 & 720,8 & 720,8 & 720,8 & 720,8 & 720,8 \\
G2 & 404 & 456 & 345 & 477 & 430 & 411 \\
G3 & 4,90 & 5,55 & 4,80 & 5,41 & 5,23 & 5,11 \\
\hline
\end{tabular}

TABELA 3 - Produtividades estimadas e observadas nas diferentes parcelas experimentais, considerando os parâmetros genéticos do híbrido A2345 próprios a cada parcela.

\begin{tabular}{ccccc}
\hline & Estimado $\left(\mathbf{k g ~ h a}^{\mathbf{- 1}}\right)$ & Observado $(\mathbf{k g ~ h a}$ \\
& $-\mathbf{1})$ & Erro \% & Diferença \\
\hline Área Total & 2177 & 4764 & 54,3 & -2587 \\
Parcela A (> teor de V\%) & 2270 & 4995 & 54,6 & -2725 \\
Parcela B (< teor de V\%) & 2122 & 4549 & 53,4 & -2427 \\
Parcela 1 (> teor de V\%) & 2000 & 4368 & 54,2 & -2368 \\
Parcela 2 (> teor de V\%) & 2539 & 5662 & 55,2 & -3123 \\
Parcela 3 (< teor de V\%) & 1706 & 3790 & 55,0 & -2084 \\
Parcela 4 (< teor de V\%) & 2551 & 5327 & 52,1 & -2776 \\
\hline
\end{tabular}


TABELA 4 - Calibração de parâmetros genéticos do híbrido A2345 para as condições experimentais.

\begin{tabular}{ccccc}
\hline Parâmetro genético & Valores tentativos & Valor simulado & Diferença & Erro (\%) \\
\hline P1 & 310 & 67 & 1 & - \\
P5 & 850,8 & 120 & 0 & - \\
G2 & 670 & 4676 & 88 & 1,85 \\
G3 & 5,19 & 4722 & 42 & 0,88 \\
\hline
\end{tabular}

TABELA 5 - Produtividades estimadas e observadas nas diferentes parcelas experimentais, considerando os valores médios dos parâmetros genéticos do híbrido A2345.

\begin{tabular}{ccccc}
\hline & Estimado $\left(\mathbf{k g ~ h a}^{-\mathbf{1}}\right)$ & Observado $\left(\mathbf{k g ~ h a}^{-\mathbf{1}}\right)$ & Erro \% & Diferença \\
\hline Área Total & 4722 & 4764 & 0,9 & -42 \\
Parcela A (> teor de V\%) & 4735 & 4995 & 5,2 & -260 \\
Parcela B (< teor de V\%) & 4717 & 4549 & 3,7 & +168 \\
Parcela 1 (> teor de V\%) & 4709 & 4368 & 7,8 & +341 \\
Parcela 2 (> teor de V\%) & 4748 & 5662 & 16,1 & -914 \\
Parcela 3 (< teor de V\%) & 4673 & 3790 & 23,3 & +883 \\
Parcela 4 (< teor de V\%) & 4732 & 5327 & 11,2 & -595 \\
\hline
\end{tabular}

TABELA 6 - Relação de valores dos parâmetros genéticos correspondentes às diferentes parcelas experimentais, obtidos após à calibração de P1, P2 e P5.

\begin{tabular}{ccccccc}
\hline Parâmetros & Parcela & Parcela2 & Parcela3 & Parcela 4 & Parcela A & Parcela \\
\hline P1 & 310 & 310 & 310 & 310 & 310 & 310 \\
P2 & 0,5 & 0,5 & 0,5 & 0,5 & 0,5 & 0,5 \\
P5 & 850,8 & 850,8 & 850,8 & 850,8 & 850,8 & 850,8 \\
G2 & 404 & 456 & 345 & 477 & 430 & 411 \\
G3 & 4,90 & 5,55 & 4,80 & 5,41 & 5,23 & 5,11 \\
\hline
\end{tabular}

Esta calibração, de forma contrária à anterior, realizou-se mantendo os parâmetros G2 e G3 sem alterar. Isto foi feito dado que calibrações realizadas até as produtividades ficarem próximas daquelas realmente obtidas, não mostrariam simulações diferentes às obtidas na calibração. Assim, calibrações realizadas para esses parâmetros poderão ser testadas somente com valores de produtividade obtidos em safras seguintes.

Pelo antes mencionado, as produtividades simuladas mostraram-se distantes das realmente observadas (Tabela 7), embora evidenciando a tendência dos valores estimados para as parcelas A e B, e para as demais parcelas. Observa-se também, a eliminação da influência negativa nas simulações nos erros similares obtidos para os dados estimados e observados.

Desta maneira, a tendência observada evidencia que, com amostragens apropriadas em locais com presença de variabilidade espacial, os modelos do DSSAT podem estimar adequadamente produtividades variáveis, coincidindo com Fraisse et al. (2001), Irmak et al. (2001) e Paz et al. (2001). 
TABELA 7 - Produtividades estimadas e observadas nas diferentes áreas experimentais posterior à calibração dos parâmetros genéticos P1, P2 e P5.

\begin{tabular}{ccccc}
\hline & Estimado $\left(\mathbf{k g ~ h a}^{\mathbf{- 1}}\right)$ & Observado $\left(\mathbf{k g ~ h a}^{-\mathbf{1}}\right)$ & Erro \% & Diferença \\
\hline Área Total & 3127 & 4764 & 34,4 & -1637 \\
Parcela A (> teor de V\%) & 3256 & 4995 & 34,8 & -1739 \\
Parcela B (< teor de V\%) & 3049 & 4549 & 33,0 & -1500 \\
Parcela 1 (> teor de V\%) & 2874 & 4368 & 34,2 & -1494 \\
Parcela 2 (> teor de V\%) & 3642 & 5662 & 35,7 & -2020 \\
Parcela 3 (< teor de V\%) & 2449 & 3790 & 35,4 & -1341 \\
Parcela 4 (< teor de V\%) & 3676 & 5327 & 31,0 & -1651 \\
\hline
\end{tabular}

\section{CONCLUSÃO}

O modelo CERES-maize foi menos eficiente em mostrar a tendência da estimativa em zonas diferenciadas segundo o atributo saturação por bases, quando utilizados os valores médios dos parâmetros genéticos.

Os parâmetros genéticos G2 e G3 apresentam variações na área experimental, evidenciando diferentes proporções nas respostas estimadas e observadas, quando considerados homogêneos.

O estudo evidencia a importância da utilização dos valores dos parâmetros de solo, manejo e genéticos próprios a cada parcela experimental, na obtenção de estimativas mais confiáveis para as simulações da cultura de milho.

\section{REFERÊNCIAS BIBLIOGRÁFICAS}

ANDRADE, F. H.; OTEGUI, M. E.; VEGA, C. Intercepted radiation at flowering and kernel number in maize. Agronomy Journal, Madison, v. 92, p. 92-97, 2000.

BATCHELOR, W. D.; BASSO, B.; PAZ, J. O. Examples of strategies to analyze spatial and temporal yield variability using crop models. European Journal of Agronomy, [S.l.], v. 18 n. 1/2, p. 141-158, 2002.

COMISSÃO DE FERTILIDADE DO SOLO DE ESTADO DE MINAS GERAIS. Recomendações para o uso de corretivos e fertilizantes em Minas Gerais: $5^{\text {a }}$ aproximação. Viçosa, 1999. 359 p.

FANCELLI, A. L. Milho: ambiente e produtividade. In: FANCELLI, A. L.; DOURADO-NETO, D. (Eds.). Milho: estratégias de manejo para alta produtividade. Piracicaba: ESALQ, 2003. p. 174-197.
FRAISSE, C. W.; SUDDUTH, K. A.; KITCHEN, N. R. Calibration of the CERES-Maize model for simulating sitespecific crop development and yield on claypan soils. Applied Engineering in Agriculture, [S.1.], v. 17, n. 4, p. 547-556, 2001.

IRMAK, A. et al. Estimating spatially variable soil properties for application of crop models in precision farming. Transaction of the ASAE, [S.1.], v. 44, n. 5, p. 13431353, 2001.

JAME, Y. W.; CURTFOURTH, H. W. Crop growth models for decision support systems. Canadian Journal of Plant Science, Ottawa, v. 76, n. 1, p. 9-19, 1996.

JONES, C. A.; KINIRY, J. R. CERES-Maize: a simulation model of maize growth and development College Station. Texas: A \& M University, 1986. 194 p.

KINIRY, J. R. et al. Evaluation of two maize models for nine U.S. locations. Agronomy Journal, Madison, v. 89, p. 421426, 1997.

LIMA, M. G. de. Calibração e validação do modelo CERESMaize em condições tropicais do Brasil. 1995. 108 f. Tese (Doutorado em Agronomia) - Escola Superior de Agricultura de Luiz de Queiroz, Piracicaba, 1995.

NIELSEN, D. C. et al. Simulating soybean water stress effects with RZWQM and CROPGRO models. Agronomy Journal, Madison, v. 94, p. 1234-1243, 2002.

PAZ, J. O. et al. Modeling approach to quantify the effects of spatial soybean yield limiting factors. Transaction of the ASAE, [S.1.], v. 44, n. 5, p. 1329-1334, 2001. 
RIBEIRO, P. H. E. Adaptabilidade e estabilidade de cultivares de milho em diferentes épocas de semeadura, níveis de adubação e locais do estado de Minas Gerais. 1998. 126 f. Tese (Doutorado em Genética e Melhoramento de Plantas) - Universidade Federal de Lavras, Lavras, 1998.

SOLER, C. T. Uso do modelo CERES-Maize para identificacao de caracteristicas genéticas desejáveis para milho "safrinha" e determinação de práticas adequadas de manejo em condicoes de risco climático. 2000. 86 f. Dissertação (Mestrado em Agronomia) Escola Superior de Agricultura de Luiz de Queiroz, Piracicaba, 2000.

XIE, Y. Maize and sorghum simulations with CERES-Maize, SORKAM, and ALMANAC under Water-Limiting conditions. Agronomy Journal, Madison, v. 93, p. 11481155, 2001.

Ciênc. agrotec., Lavras, v. 29, n. 6, p. 1153-1160, nov./dez., 2005 